\title{
Evaluation of the drug-induced lymphocyte stimulation test for diagnosing mesalazine allergy
}

\author{
Daisuke Saito, Mari Hayashida, Taro Sato, Shintaro Minowa, Osamu Ikezaki, Tatsuya Mitsui, Miki Miura, \\ Akihito Sakuraba, Tadakazu Hisamatsu \\ The Third Department of Internal Medicine, Kyorin University School of Medicine, Mitaka, Japan
}

\begin{abstract}
Background/Aims: Mesalazine is an effective drug for treating ulcerative colitis (UC), but causes allergic symptoms in a few cases. Therefore, the objective of this study was to evaluate the usefulness of the drug-induced lymphocyte stimulation test (DLST) for the diagnosis of mesalazine allergy. Methods: Patients with UC treated with mesalazine with or without a history of associated adverse events (AEs) were enrolled at Kyorin University Hospital from July 2016 to April 2017. Results: The DLST was performed in 104 patients with UC, of which 24 had a history of AEs due to mesalazine treatment. The control value of DLST was $337.4 \pm 296.3$ counts per minute $(\mathrm{cpm})$ in the $\mathrm{AE}^{+}$group and $408.0 \pm 371.9 \mathrm{cpm}$ in the $\mathrm{AE}^{-}$group. The measured value of DLST was $578.8 \pm 424.7 \mathrm{cpm}$ in the $\mathrm{AE}^{+}$group and $476.5 \pm 471.8 \mathrm{cpm}$ in the $\mathrm{AE}^{-}$group. The stimulation index (SI) was $243.9 \% \pm 291.1 \%$ in the $\mathrm{AE}^{+}$group and $119.8 \% \pm 53.0 \%$ in the $\mathrm{AE}^{-}$group. The SI value and DLST positivity were significantly higher in the $\mathrm{AE}^{+}$group than in the $\mathrm{AE}^{-}$group $(P=0.030$ and $P=0.029$, respectively). The test sensitivity and specificity were 0.240 and 0.805 , respectively, and the false-positive and false-negative rate was 0.195 and 0.760 , respectively. Conclusions: The DLST for mesalazine showed low sensitivity and high specificity, suggesting that it may be useful for the definitive diagnosis of allergy to mesalazine. (Intest Res 2018;16:273-281)
\end{abstract}

Key Words: Mesalamine; Drug-induced lymphocyte stimulation test; Colitis, ulcerative

\section{INTRODUCTION}

The incidence of UC is increasing worldwide and is currently estimated at more than 180,000 cases in Japan. ${ }^{1}$ The first-line drug for treatment of mild to moderate UC is 5-aminosalicylate (5-ASA), and most UC patients with mild to moderate UC activity can achieve disease remission only with 5-ASA therapy. ${ }^{2,3}$ Furthermore, the continuous use of 5-ASA contributes to the maintenance of remission. ${ }^{2,4}$ Mesalazine was developed as a formulation free from sulfapyridine, which is a salazosulfapyridine (SASP) component that produces side effects. ${ }^{5}$ Because the effect of 5-ASA de-

Received August 11, 2017. Revised October 7, 2017.

Accepted October 8, 2017. Published online December 1, 2017

Correspondence to Tadakazu Hisamatsu, The Third Department of Internal

Medicine, Kyorin University School of Medicine, 6-20-2 Shinkawa, Mitaka

181-8611, Japan. Tel: +81-422-47-5511 (ext. 5279), Fax: +81-422-71-5381,

E-mail: thisamatsu@ks.kyorin-u.ac.jp pends on its concentration in the colonic mucosa, a system for drug delivery to the large intestine is necessary for each 5-ASA preparation. In Japan, Pentasa ${ }^{\circledR}$, a time- and moisturedependent release system, and Asacol ${ }^{\circledR}$, a pH-dependent, colon-targeted oral drug delivery system, are available, and the multi-matrix system Lialda ${ }^{\circledR}$ has recently been approved. However, the occurrence of allergic reactions to mesalazine is clear, and symptoms include fever, abdominal pain, diarrhea, and hematochezia. ${ }^{6-8}$ These symptoms may often resemble exacerbations of UC activity and represent a limitation of mesalazine use in routine clinical practice. However, mesalazine allergy has been reported only in case series, and the actual condition has not been clarified to date.

The drug-induced lymphocyte stimulation test (DLST) is commonly used for auxiliary diagnosis of drug allergies ${ }^{9}$ and measures ${ }^{3} \mathrm{H}$-thymidine uptake by proliferating lymphocytes following stimulation with the drug of interest. However, the DLST produces false-positive and false-negative results and

๑ Copyright 2018. Korean Association for the Study of Intestinal Diseases. All rights reserved.

This is an Open Access article distributed under the terms of the Creative Commons Attribution Non-Commercial License (http://creativecommons.org/licenses/by-nc/4.0)

which permits unrestricted non-commercial use, distribution, and reproduction in any medium, provided the original work is properly cited. 
its value in the diagnosis of drug allergies appears to vary greatly depending on the drug of interest. ${ }^{10-13}$ For these reasons, the diagnosis of drug allergies often involves the analysis of the clinical course, in addition to the DLST results. Few studies to date have evaluated the sensitivity and specificity of DLST in the diagnosis of allergy to mesalazine, and thus the usefulness of this test in this context remains unclear. Mesalazine is well established as maintenance therapy for UC and may prevent the development of colorectal cancer. ${ }^{14}$ The use of a mesalazine sustained-release system in the treatment of UC may help improve disease management over the lifetime of a patient. For this reason, accurate diagnosis of allergy to mesalazine is an important aspect of the treatment of UC.

In this study, the clinical features of UC patients with mesalazine allergy were investigated, and DLST was conducted in UC patients with and without adverse events (AEs) caused by mesalazine treatment to evaluate the usefulness of DLST for the diagnosis of these allergies.

\section{METHODS}

\section{Drug-Induced Lymphocyte Stimulation Test}

DLST was performed by SRL, Inc. (Hachioji, Japan).

Briefly, $20 \mathrm{~mL}$ of whole blood was collected from each patient and transferred to heparinized blood collection tubes
(SRL, Inc.). Lymphocytes isolated from whole blood by specific gravity centrifugation were suspended in RPMI1640 culture medium (DS Pharma Biomedical, Osaka, Japan) and incubated with mesalazine for 72 hours. Next, ${ }^{3} \mathrm{H}$-thymidine was added to the medium and the cells were incubated for 16 hours. ${ }^{3} \mathrm{H}$-thymidine uptake by lymphocytes not treated with mesalazine was used as a control. The ratio of ${ }^{3} \mathrm{H}$-thymidine uptake between the mesalazine-treated and control samples was set as the stimulation index (SI). A positive DLST result was defined as an SI $>180 \%$.

\section{Patients}

This study complied with ethical principles established in the Declaration of Helsinki, the Ministerial Ordinance on Good Clinical Practice for Drugs, and other relevant laws, regulations, and standards. Written informed consent was obtained from each patient. The study protocol, sample case report form, patient information sheet, and informed consent form were approved by the Research Ethics Committee of Kyorin University Medical School (approval number: 745) before the initiation of the study; the design of the clinical trial was also approved.

A total of 105 patients with UC who were treated at Kyorin University Hospital from July 2016 to April 2017 were enrolled in the study and underwent the DLST. Patients receiving corticosteroids, patients with a history of total colectomy,

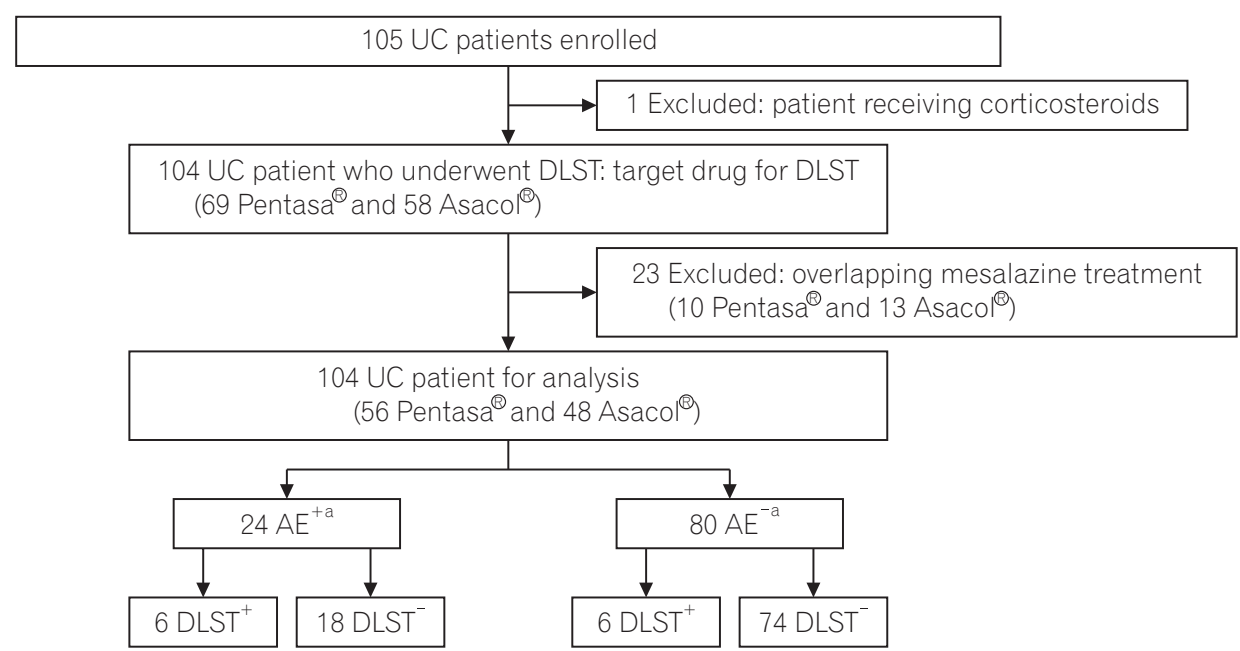

Fig. 1. Flow chart of the study protocol. Drug-induced lymphocyte stimulation test (DLST) was performed in 105 patients receiving mesalazine without side effects or having previously received mesalazine with adverse events (AEs). Patients receiving corticosteroids, patients with a history of total colectomy, and patients who could not provide informed consent were excluded $(n=1)$. Where there was a history of AE associated with 2 types of mesalazine preparation in the same patient, DLST was evaluated for the first preparation to cause AE and overlapping was excluded (10 Pentasa ${ }^{\circledR}$ and 13 Asacol ${ }^{\circledR}$ ). DLST positivity was 6 out of 24 cases in $\mathrm{AE}^{+}$(with AEs) and 6 out of 80 cases in $\mathrm{AE}^{-}$(without AEs) patients. ${ }^{\mathrm{a}} \mathrm{AEs}$ were defined as symptoms that appeared within 30 days of the first administration of mesalazine and disappeared after discontinuation of mesalazine. 
and patients who did not provide informed consent were excluded. AEs were defined as symptoms that appeared within 30 days of the first administration of mesalazine and disappeared after discontinuation of mesalazine. The AEs that required hospitalization or treatment other than discontinuation of mesalazine were defined as severe AEs (SAEs). Patients either received mesalazine during the study period without side effects or had previously received mesalazine and developed AEs. In cases in which a history of AEs was associated with a combined mesalazine preparation (10 Pentasa ${ }^{\circledR}$ and 13 Asacol $^{\circledR}$ ) in the same patient, the DLST using the drug administered first was considered and the DLSTs using the combined preparation were excluded. Information on AEs was obtained from the medical records, and symptoms that appeared within 30 days after the first administration of mesalazine and disappeared after discontinuation of mesalazine was considered evidence of allergic

Table 1. Clinical Characteristics of Patients at Baseline $(n=104)$

\begin{tabular}{|c|c|c|c|}
\hline Characteristics & $\mathrm{AE}^{+}(\mathrm{n}=24)$ & $\mathrm{AE}^{-}(\mathrm{n}=80)$ & $P$-value \\
\hline Age (yr) & $36.1 \pm 14.4$ & $45.9 \pm 13.6$ & $0.062^{\mathrm{a}}$ \\
\hline Sex & & & $0.242^{b}$ \\
\hline Male & 9 & 43 & \\
\hline Female & 15 & 37 & \\
\hline \multicolumn{4}{|l|}{ Extent of colitis } \\
\hline Pancolitis & 13 & 40 & $0.817^{b}$ \\
\hline Left-sided colitis & 9 & 24 & $0.618^{b}$ \\
\hline Proctitis & 2 & 16 & $0.233^{b}$ \\
\hline \multicolumn{4}{|l|}{ Severity classification } \\
\hline Mild & 21 & 77 & $0.134^{b}$ \\
\hline Moderate & 3 & 3 & $0.134^{b}$ \\
\hline Duration of disease (mo) & $76.2 \pm 90.2$ & $95.1 \pm 82.6$ & $0.318^{\mathrm{a}}$ \\
\hline \multicolumn{4}{|l|}{ 5-ASA agent } \\
\hline Pentasa ${ }^{\circledR}$ & 2 & 46 & $<0.001^{b}$ \\
\hline Asacol ${ }^{\circledR}$ & 1 & 33 & $<0.001^{b}$ \\
\hline SASP & 8 & 2 & $<0.001^{b}$ \\
\hline \multicolumn{4}{|l|}{ Concomitant medications } \\
\hline AZA & 8 & 10 & $0.291^{b}$ \\
\hline IFX & 0 & 8 & $0.193^{b}$ \\
\hline ADA & 1 & 1 & $0.410^{b}$ \\
\hline
\end{tabular}

Values are presented as mean \pm SD or number.

aMann-Whitney test.

${ }^{b}$ Fisher exact test.

$A E$, adverse event; $A E^{+}$, patients with $A E s ; A E^{-}$, patients without $A E s$; 5-ASA, 5-aminosalicylate; SASP, salazosulfapyridine; AZA, azathioprine; IFX, infliximab; ADA, adalimumab.
AEs. A flowchart of the study protocol is shown in Fig. 1.

\section{Statistical Analysis}

Statistical analysis was conducted using JMP ${ }^{\circledR}$ software version 10 (SAS Institute Inc., Cary, NC, USA). Categorical variables were analyzed using Fisher exact test, and continuous variables were analyzed using the Mann-Whitney test. $P$-values of less than 0.05 were considered statistically significant.

\section{RESULTS}

\section{Patient Characteristics}

DLST was performed on 104 patients (mean age, $40.7 \pm 14.6$ years; 52 men and 52 women). Patients' baseline and clinical characteristics are shown in Table 1. DLST was performed in 24 patients with a history of AEs ( $\mathrm{AE}^{+}$group) and 80 patients without $\mathrm{AEs}$ ( $\mathrm{AE}^{-}$group). With regard to patient characteristics, there were no significant differences between the 2 groups except for the type of 5-ASA preparation used.

Table 2. Results of the DLST for Mesalazine

\begin{tabular}{lccc}
\hline & $\mathrm{AE}^{+}(\mathrm{n}=\mathbf{2 4})$ & $\mathrm{AE}^{-}(\mathrm{n}=\mathbf{8 0})$ & $\boldsymbol{P}$-value \\
\hline Control value $(\mathrm{cpm})$ & $337.4 \pm 296.3$ & $408.0 \pm 371.9$ & $0.302^{\mathrm{a}}$ \\
Measured value $(\mathrm{cpm})$ & $578.8 \pm 424.7$ & $476.5 \pm 471.8$ & $0.275^{\mathrm{a}}$ \\
Stimulation index $(\%)$ & $243.9 \pm 291.1$ & $119.8 \pm 53.0$ & $0.030^{\mathrm{a}}$ \\
Positive DLST & 6 & 6 & $0.029^{\mathrm{c}}$ \\
Negative DLST & 18 & 74 & $0.029^{\mathrm{c}}$ \\
\hline
\end{tabular}

Values are presented as mean \pm SD or number

aMann-Whitney test.

${ }^{b}$ DLST positivity was defined as a stimulation index $>180 \%$.

${ }^{\mathrm{c}}$ Fisher exact test.

DLST, drug-induced lymphocyte stimulation test; $A E_{\text {, adverse event; }}$ cpm, counts per minute.

Table 3. Sensitivity and Specificity of the DLST for Mesalazine Allergy

\begin{tabular}{ll}
\hline & Value \\
\hline Sensitivity & 0.240 \\
Specificity & 0.805 \\
False-positive rate & 0.195 \\
False-negative rate & 0.760 \\
\hline
\end{tabular}

DLST, drug-induced lymphocyte stimulation test. 
Table 4. Symptoms Associated with Adverse Events Following Mesalazine Treatment ( $n=24)$

\begin{tabular}{|c|c|c|c|c|c|c|c|}
\hline Case & Watery diarrhea & High fever & Hematochezia & Skin eruptions & Abdominal pain & Liver damage & Pneumonitis \\
\hline 1 & (a) & (a) & & & & & \\
\hline 2 & $\bigcirc$ & $\bigcirc$ & & & $\bigcirc$ & & \\
\hline 3 & & & & () 0 & & & \\
\hline 4 & & & & & & O & \\
\hline 5 & & & & (a) 0 & & & \\
\hline 6 & & & & & & & 0 \\
\hline 7 & 0 & $\bigcirc$ & & & & & \\
\hline 8 & & & & (a) 0 & & & \\
\hline 9 & & & & & & (a) & \\
\hline 10 & 0 & & 0 & & & & \\
\hline 11 & & & & & $\bigcirc$ & & \\
\hline 12 & (a) & (a) & & & & & (0) \\
\hline 13 & $\bigcirc$ & & $\bigcirc$ & & & & \\
\hline 14 & (C) & (C) & & & & & \\
\hline 15 & $\bigcirc$ & $\bigcirc$ & O & & & & \\
\hline 16 & O & O & & & & & \\
\hline 17 & (a) & (a) & & & () & & \\
\hline 18 & (C) & (a) & $\bigcirc$ & & & & \\
\hline 19 & $\bigcirc$ & & 0 & & & & \\
\hline 20 & (0) & & & & & & \\
\hline 21 & (C) & & & & & & \\
\hline 22 & & & & 0 & & & \\
\hline 23 & (a) & & & & & & \\
\hline 24 & & & (2) & & & & \\
\hline
\end{tabular}

\section{Drug-Induced Lymphocyte Stimulation Test}

The control value of DLST was $337.4 \pm 296.3$ counts per minute $(\mathrm{cpm})$ in the $\mathrm{AE}^{+}$group and $408.0 \pm 371.9 \mathrm{cpm}$ in the $\mathrm{AE}^{-}$group. The measured value of DLST was $578.8 \pm 424.7$ cpm in the $\mathrm{AE}^{+}$group and $476.5 \pm 471.8 \mathrm{cpm}$ in the $\mathrm{AE}^{-}$group. The SI was significantly higher in the $\mathrm{AE}^{+}$group than in the $\mathrm{AE}^{-}$group $(243.9 \% \pm 291.1 \%$ and $119.8 \% \pm 53.0 \%$, respectively, $P=0.030$ ). DLST positivity was significantly higher in the $\mathrm{AE}^{+}$ group than in the $\mathrm{AE}^{-}$group (6/24 [25.0\%] and 6/80 [7.5\%], respectively, $P=0.029$ ) (Table 2 ). The sensitivity and specificity of DLST were 0.240 and 0.805 , respectively, and the false-positive and false-negative rate was 0.195 and 0.760 , respectively (Table 3 ). The interval from the time of onset of allergic symptoms to the time of execution of the DLST was 2 weeks in 3 cases, $\leq 1$ month in 2 cases, and $>1$ month in 19 cases. DLST positivity was 1 of $3(33.3 \%)$ within 2 weeks, 0 of
Table 5. Relationship between Timing of Drug Administration and Onset of Adverse Events

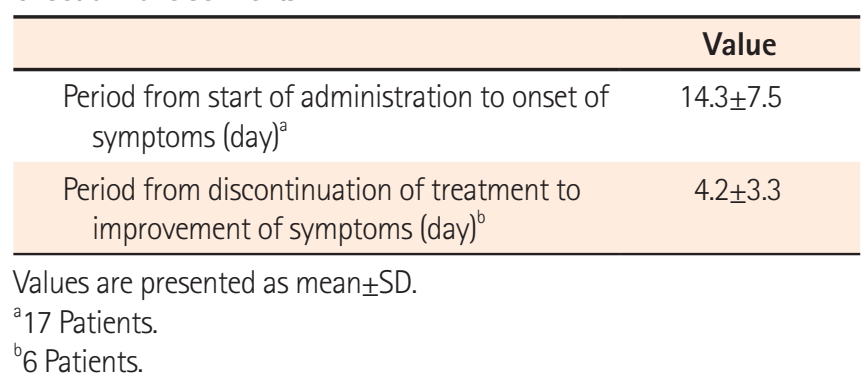

$2(0 \%)$ within 1 month, and 5 of $19(26.3 \%)$ after 1 month.

\section{AEs Associated with Mesalazine Treatment}

The reported AEs were watery diarrhea $(n=15)$, high fever $(n=9)$, hematochezia $(n=6)$, abdominal pain $(n=3)$, skin 
eruptions ( $n=3)$, pneumonitis ( $n=2)$, and liver damage $(n=2)$ (some of these events overlapped). In 4 patients, symptoms appeared after the administration of both Asacol ${ }^{\circledR}$ and Pen$\operatorname{tasa}^{\circledR}$ formulations (Table 4). The mean period from the start of administration to symptom onset was $14.3 \pm 7.5$ days. The mean period from treatment discontinuation to symptom improvement was $4.2 \pm 3.3$ days (Table 5). Among the 24 patients with AEs, 5 cases of SAE were reported. The clinical characteristics of patients with SAEs $\left(\mathrm{SAE}^{+}\right)$are shown in Table 6. There was no significant difference $(P=0.643)$ in the average number of days to onset of SAEs relative to the onset of AEs that were not SAEs, or in the average number of days to improvement of symptoms $(P=0.863)$. Table 7 shows the DLST results for $\mathrm{SAE}^{+}$and $\mathrm{SAE}^{-}$cases among all $\mathrm{AE}^{+}$cases. DLST positivity was $40.0 \%$ in $\mathrm{SAE}^{+}$cases and $26.3 \%$ in $\mathrm{SAE}^{-}$ cases. Many AE symptoms overlapped, which limited the evaluation of each symptom. However, the most common combination of symptoms was high fever and watery diar- rhea. Nine patients presented these 2 symptoms, and DLST was positive in 3 of these patients (30.0\%). The clinical characteristics of patients with both high fever and diarrhea are shown in Table 8. A comparison between the DLST-positive and DLST-negative group indicated that the number of AEs

Table 7. Results of the DLST in UC patients with or without SAEs

\begin{tabular}{lcc}
\hline & $\mathrm{AE}^{+} / \mathrm{SAE}^{-}(\mathrm{n}=19)$ & $\mathrm{AE}^{+} / \mathrm{SAE}^{+}(\mathrm{n}=5)$ \\
\hline Control value $(\mathrm{cpm})$ & $320.4 \pm 256.3$ & $323.4 \pm 293.4$ \\
Measured value $(\mathrm{cpm})$ & $518.8 \pm 312.1$ & $713.2 \pm 236.5$ \\
Stimulation index $(\%)$ & $227.1 \pm 183.6$ & $421.9 \pm 201.3$ \\
Positive DLST & 4 & 2 \\
Negative DLST & 15 & 3 \\
\hline
\end{tabular}

Values are presented as mean $\pm S D$ or number.

DLST, drug-induced lymphocyte stimulation test; SAE, severe adverse event; $A E$, adverse event; $c p m$, counts per minute.

Table 6. Clinical Characteristics of UC Patients with Severe Adverse Events Following Mesalazine Treatment

\begin{tabular}{|c|c|c|c|c|c|c|c|}
\hline No. & $\begin{array}{l}\text { Age } \\
(y r)\end{array}$ & Sex & Symptoms & $\begin{array}{l}\text { Period from start of } \\
\text { administration to onset } \\
\text { of symptoms (day) }\end{array}$ & $\begin{array}{c}\text { Period from } \\
\text { discontinuation of } \\
\text { mesalazine to improvement } \\
\text { of symptoms (day) }\end{array}$ & $\begin{array}{l}\text { Treatment } \\
\text { for UC after } \\
\text { discontinuation } \\
\text { of mesalazine }\end{array}$ & $\begin{array}{l}\text { DLST } \\
\text { result }\end{array}$ \\
\hline 1 & 45 & $\mathrm{~F}$ & High fever, abdominal pain & 11 & 5 & Probiotics & Negative \\
\hline 2 & 19 & $\mathrm{~F}$ & Pneumonia & 18 & 4 & Probiotics, AZA & Positive \\
\hline 3 & 33 & $\mathrm{~F}$ & Watery diarrhea, hematochezia & 10 & 4 & Probiotics, SASP & Negative \\
\hline 4 & 14 & M & $\begin{array}{l}\text { High fever, watery diarrhea, } \\
\text { pneumonitis }\end{array}$ & 20 & 4 & Probiotics, AZA & Positive \\
\hline 5 & 21 & $\mathrm{~F}$ & High fever, watery diarrhea & Unknown & Unknown & Probiotics & Negative \\
\hline
\end{tabular}

DLST, drug-induced lymphocyte stimulation test; F, female; AZA, azathioprine; SASP, salazosulfapyridine; M, male.

Table 8. Clinical Characteristics of UC Patients with Watery Diarrhea and High Fever

\begin{tabular}{|c|c|c|c|c|c|c|c|}
\hline No. & $\begin{array}{l}\text { Age } \\
\text { (yr) }\end{array}$ & Sex & SAE & $\begin{array}{l}\text { Period from start of } \\
\text { administration to } \\
\text { onset of symptoms (day) }\end{array}$ & $\begin{array}{l}\text { Period from discontinuation of } \\
\text { mesalazine to improvement of } \\
\text { symptoms (day) }\end{array}$ & $\begin{array}{c}\text { Treatment for UC } \\
\text { after discontinuation of } \\
\text { mesalazine }\end{array}$ & $\begin{array}{l}\text { DLST } \\
\text { results }\end{array}$ \\
\hline 1 & 21 & M & - & 10 & 8 & Probiotics & Positive \\
\hline 2 & 45 & $\mathrm{~F}$ & + & 11 & 5 & Probiotics & Negative \\
\hline 3 & 18 & M & - & Unknown & Unknown & Probiotics, AZA & Negative \\
\hline 4 & 14 & M & + & 20 & 4 & Probiotics, AZA & Positive \\
\hline 5 & 21 & $\mathrm{~F}$ & + & Unknown & Unknown & Probiotics & Negative \\
\hline 6 & 17 & M & - & Unknown & Unknown & Probiotics, AZA, SASP & Negative \\
\hline 7 & 17 & $\mathrm{~F}$ & - & Unknown & Unknown & Probiotics & Negative \\
\hline 8 & 46 & $F$ & - & 7 & 3 & Probiotics, AZA, SASP & Negative \\
\hline 9 & 47 & M & - & Unknown & Unknown & Probiotics & Positive \\
\hline
\end{tabular}

SAE, severe adverse event; DLST, drug-induced lymphocyte stimulation test; $M$, male; F, female; AZA, azathioprine; SASP, salazosulfapyridine. 
Table 9. Clinical Characteristics of UC Patients with Positive or Negative DLST Results

\begin{tabular}{|c|c|c|c|}
\hline Characteristics & $\begin{array}{l}\text { Positive DLST } \\
\qquad(n=12)\end{array}$ & $\begin{array}{l}\text { Negative DLST } \\
\quad(n=92)\end{array}$ & $P$-value \\
\hline Age (yr) & $40.3 \pm 13.2$ & $42.6 \pm 14.2$ & $0.182^{a}$ \\
\hline Sex & & & $0.358^{b}$ \\
\hline Male & 4 & 48 & \\
\hline Female & 8 & 44 & \\
\hline \multicolumn{4}{|l|}{ Extent of colitis } \\
\hline Pancolitis & 9 & 44 & $0.123^{b}$ \\
\hline Left-sided colitis & 2 & 31 & $0.332^{b}$ \\
\hline Proctitis & 1 & 17 & $0.687^{b}$ \\
\hline \multicolumn{4}{|l|}{ Severity classification } \\
\hline Mild & 10 & 88 & $0.141^{b}$ \\
\hline Moderate & 2 & 4 & $0.141^{b}$ \\
\hline Duration of disease (mo) & $71.6 \pm 66.3$ & $98.2 \pm 89.8$ & $0.397^{\mathrm{a}}$ \\
\hline$A E$ & 6 & 18 & $0.029^{b}$ \\
\hline SAE & 2 & 3 & $0.101^{b}$ \\
\hline
\end{tabular}

Values are presented as mean \pm SD or number.

${ }^{a}$ Mann-Whitney test.

${ }^{b}$ Fisher exact test.

DLST, drug-induced lymphocyte stimulation test; $A E$, adverse event; $S A E$, severe $A E$.

was significantly higher in the DLST-positive group; nonetheless, no other significant item was found in other patient backgrounds (Table 9).

\section{DISCUSSION}

A previous study found a DLST positivity of approximately $40 \%,{ }^{15}$ although results may differ depending on the target drug, indicating that DLST evaluation should be based on the results obtained for a given drug. Mesalazine is effective for the treatment of IBD, including UC. However, this drug has been reported to cause allergies in a few cases, and the DLST is frequently used for the auxiliary diagnosis of mesalazine allergy. However, the sensitivity and specificity of the DLST for the diagnosis of mesalazine allergy have not been evaluated to date. To the best of our knowledge, this study is the first to thoroughly evaluate DLST results for mesalazine allergy.

Our results revealed that the DLST had low sensitivity and high specificity in patients with suspected mesalazine allergy, suggesting that the DLST might be suitable for the definitive diagnosis but not the exclusive diagnosis of mesalazine allergy. With respect to the usefulness of the DLST for the diagnosis of drug allergies, the test results differ depending on the properties of the drug of interest, and the reliability varies significantly according to the drug. For example, the DLST for acetaminophen, NSAID, and Chinese medicine has shown high positivity. ${ }^{16}$ However, Chinese medicine exerts an immunopotentiating effect and may produce falsepositive DLST results. ${ }^{17}$ In addition, anti-cytotoxic drugs with strong cytotoxicity are diluted before being tested because of their toxicity even in vitro and consequently produce a low DLST positivity. However, antimetabolites that inhibit DNA synthesis, such as 5-fluorouracil, activate the salvage pathway, a DNA recycling mechanism, ${ }^{13,17}$ and cells exposed to these agents showed increased ${ }^{3} \mathrm{H}$-thymidine uptake with high cpm values, even though the examined cells were not proliferating. In addition, vancomycin, chlorella, $\beta$-lactam antibiotics, and some contrast agents, among others, can cause nonspecific lymphocyte stimulation in vitro. ${ }^{18}$ The DLST positivity for mesalazine is considered to fall into the category of common drugs ${ }^{13}$ and does not show very high or very low values, indicating that the DLST can be used for auxiliary diagnosis of mesalazine allergy. Therefore, it appears that the diagnosis of these allergies should involve the analysis of the clinical course, and DLST can be useful for auxiliary diagnosis.

Various symptoms of mesalazine allergy have been reported. Although few studies have evaluated these symptoms in detail, Hanauer et al. ${ }^{6}$ reported that the incidence of diarrhea was $4.6 \%$ and the incidence of hematochezia and fever was $1.4 \%$. Shimizu et al. ${ }^{19}$ reported that, out of 88 pediatric patients with UC, 11 patients presented allergy to 5-ASA, and the DLST positivity rate was $80 \%$, which is higher than the rate observed in our study. However, differences in the timing and method of the DLST and the observed allergic symptoms may contribute to differences in DLST positivity between studies. In addition, although the DLST positivity has not been compared between pediatric and adult IBD patients to date, positivity may differ according to age.

Previous studies have shown that watery diarrhea and fever are typical symptoms of mesalazine allergy, which is consistent with the results of our study. These symptoms combined were observed in 3 out of 9 cases (30\%). These symptoms should be differentiated from UC exacerbations by promptly diagnosing mesalazine allergy and discontinuing treatment if necessary. However, even in patients with these symptoms, DLST positivity was $30 \%$ in our series and the comprehensive evaluation of allergic symptoms according to the clinical course was necessary. 
Most importantly, in many studies, DLST was performed only in patients suspected of having drug allergies. In these cases, the false-positive rate of DLST, i.e., the positive rate in patients without allergic reactions, was not evaluated. This does not imply that we correctly determined the clinical usefulness of DLST for drugs classified into general categories, such as mesalazine. The importance of DLST in the diagnosis of mesalazine allergy was determined for the first time by conducting this test in patients without side effects. Therefore, our results indicated that DLST positivity was also observed in patients treated with mesalazine without side effects. However, even in patients suspected of having mesalazine allergy, DLST was not necessarily positive, indicating that, at least for mesalazine, DLST should not be used for allergy screening. Positivity in patients with a high suspicion of allergies has important implications for definitive diagnosis; however, the possibility of false negatives cannot be overlooked.

Furthermore, in suspected cases of mesalazine allergy, re-administration of the drug is not advised because of the high risk of allergy relapse, and for this reason, the selection of a treatment that can maintain remission is necessary. In our study, remission in many allergy cases was maintained by the administration of probiotics and azathioprine, but SASP was also administered to some patients. SASP and mesalazine preparations (Pentasa ${ }^{\circledR}$ and Asacol $^{\circledR}$ ) are classified as 5-ASA agents and share some characteristics. SASP should not be administered to patients suspected of developing mesalazine allergy. However, patients with suspected mesalazine allergy achieved and maintained remission by switching to SASP treatment. ${ }^{20}$ Compared to SASP, mesalazine preparations contain additives that may cause allergies, and thus symptoms may not develop with SASP treatment. However, in our study, allergic symptoms such as fever and watery diarrhea occurred even among patients with allergy who received SASP and, for this reason, it was difficult to predict whether SASP could be used.

SASP treatment may be an option for maintaining remission in patients suspected of having mesalazine allergy. However, careful follow-up observation is necessary because it is difficult to predict whether these patients can be treated with SASP without developing allergic symptoms. A previous study ${ }^{21}$ reported that desensitization therapy might be useful; however, this strategy was not used at our hospital.

DLST positivity for SAEs was $40 \%$, which was higher than in cases of AEs. In this respect, it is possible that the sensitivity and specificity of DLST change in severe cases compared with patients with typical symptoms. During the study pe- riod, the mesalazine preparations in use in Japan were Pen$\operatorname{tasa}^{\circledR}$ and Asacol $^{\circledR}$, and these preparations contain the same components and produce the same allergic symptoms. In our study, AEs were caused by both Pentasa ${ }^{\circledR}$ and Asacol ${ }^{\circledR}$ in 5 out of 24 cases. However, despite containing the same ingredients, these 2 drugs differ in the composition of the additives, which may lead to differences in the development of AEs. Therefore, more studies are necessary to investigate the role of additives in mesalazine preparations on the development of mesalazine allergy. Furthermore, endoscopic findings in patients with mesalazine allergy have been classified as Mayo Endoscopic subscore 1 or 2 and thus are difficult to distinguish from UC exacerbation. ${ }^{22,23}$ Endoscopic findings were not evaluated in this study and more studies are necessary to determine whether endoscopy is useful in the diagnosis of mesalazine allergy. Considering that symptoms of mesalazine allergy may be difficult to distinguish from symptoms related to UC exacerbation, delayed diagnosis of mesalazine allergy may lead to unnecessary treatment for UC. Unnecessary treatment should be avoided in UC because the criteria for discontinuation are not well-defined for many drugs, including biological preparations. In addition, misdiagnosis of mesalazine allergy may lead to unnecessary treatment discontinuation. Therefore, the accurate diagnosis of mesalazine allergy by making the appropriate interpretation of the DLST for auxiliary diagnosis is essential.

A limitation of this study was the timing of the execution of the DLST. Some studies evaluated the relationship between the time of onset of allergic symptoms and the time of the DLST. Pichler and Tilch ${ }^{24}$ and Popple et al. ${ }^{25}$ reported that, because false-positive results were increased in the acute phase, DLST should not be performed in this phase. Sugihara et al. ${ }^{26}$ compared the results of the DLST in the acute and chronic phase and reported that the sensitivity was increased in the chronic phase. However, in patients with skin eruptions, it appears that the DLST should be conducted in the acute phase.$^{27}$ Therefore, in mesalazine allergy, DLST positivity may differ according to the time of testing. In this study, DLST was performed in patients with a history of AEs, and thus there was an interval from the time of onset of allergic symptoms to the time of DLST. Consequently, DLST positivity may have differed slightly at the onset of AEs. The comparison of the DLST results between the acute and chronic phase of mesalazine allergy is necessary to confirm this hypothesis. In this study, the DLST was conducted primarily in the chronic phase of allergic symptoms. For this reason, future studies should conduct the DLST in the acute phase. 
The DLST is primarily used for the diagnosis of type IV allergy; however, T lymphocytes that recognize drug antigens in the DLST also react to other types of allergens, ${ }^{24}$ therefore, the DLST may be a helpful reference for the diagnosis of other types of allergies. In our study, the difference in the DLST prevalence rate due to symptoms did not yield significant results, but DLST positivity specific to symptoms may exist.

In our study, we excluded patients receiving steroids but not those receiving azathioprine, infliximab, or adalimumab, which are used to maintain remission by immunosuppression. The effect of these drugs on the DLST is unknown, however, these drugs may not affect the SI because they also affected the control group.

In conclusion, DLST for mesalazine showed low sensitivity and high specificity, suggesting that this test might be useful for the definitive diagnosis but not the exclusive diagnosis of mesalazine allergy. However, it is likely that the diagnosis of mesalazine allergy based solely on the DLST results is disadvantageous in the treatment of UC. Allergy to mesalazine and other drugs needs to comprehensively judge the clinical course and make a diagnosis. DLST should be used for complementary diagnosis.

\section{FINANCIAL SUPPORT}

The authors received no financial support for the research, authorship, and/or publication of this article.

\section{CONFLICT OF INTEREST}

No potential conflict of interest relevant to this article was reported.

\section{AUTHOR CONTRIBUTION}

D.S. and T.H. were involved in conception and design of the study, acquisition and analysis of data.

All authors were involved in interpretation of data, drafting and critical revision of the manuscript and have approved the final manuscript for submission. All authors also had full access to all the data in the study and had final responsibility for the decision to submit for publication.

\section{REFERENCES}

1. Matsuoka K, Watanabe M. Ulcerative colitis-recent advance in clinical practice and basic research. Nihon Shokakibyo Gakkai Zasshi 2016;113:407-412.
2. Ford AC, Achkar JP, Khan KJ, et al. Efficacy of 5-aminosalicylates in ulcerative colitis: systematic review and meta-analysis. Am J Gastroenterol 2011;106:601-616.

3. Feagan BG, Macdonald JK. Oral 5-aminosalicylic acid for induction of remission in ulcerative colitis. Cochrane Database Syst Rev 2012;10:CD000543. doi: 10.1002/14651858.CD000543. pub3.

4. Sutherland L, Macdonald JK. Oral 5-aminosalicylic acid for maintenance of remission in ulcerative colitis. Cochrane Database Syst Rev 2006;(2):CD000544. doi: 10.1002/14651858. CD000544.pub2.

5. Kobayashi K, Mukae M, Ogawa T, et al. 5-Aminosalicylic acid preparations for ulcerative colitis. Intestine 2013;17:127-132.

6. Hanauer S, Schwartz J, Robinson M, et al. Mesalamine capsules for treatment of active ulcerative colitis: results of a controlled trial. Pentasa Study Group. Am J Gastroenterol 1993;88:11881197.

7. Iofel E, Chawla A, Daum F, Markowitz J. Mesalamine intolerance mimics symptoms of active inflammatory bowel disease. J Pediatr Gastroenterol Nutr 2002;34:73-76.

8. Shimodate Y, Takanashi K, Waga E, Fujita T, Katsuki S, Nomura M. Exacerbation of bloody diarrhea as a side effect of mesalamine treatment of active ulcerative colitis. Case Rep Gastroenterol 2011;5:159-165.

9. Nyfeler B, Pichler WJ. The lymphocyte transformation test for the diagnosis of drug allergy: sensitivity and specificity. Clin Exp Allergy 1997;27:175-181.

10. Mantani N, Kogure T, Sakai S, et al. Incidence and clinical features of liver injury related to Kampo (Japanese herbal) medicine in 2,496 cases between 1979 and 1999: problems of the lymphocyte transformation test as a diagnostic method. Phytomedicine 2002;9:280-287.

11. Watanabe M, Shibuya A, Satomichi A, et al. Diagnostic value of drug lymphocyte stimulation test and evaluation of diagnostic criteria for drug induced liver injury. Kanzo 2001;42:448-454.

12. Hagiwara K, Sato T, Akiyama O. Low specificity of lymphocyte stimulation test for methotrexate in the patients with rheumatoid arthritis. Allergy Pract 2006;26:46-50.

13. Kawabata R, Koida M, Kanie S, Tanaka G, Ohuchida A, Yoshida T. DLST as a method for detecting TS-1-induced allergy. Gan To Kagaku Ryoho 2006;33:345-348.

14. Velayos FS, Terdiman JP, Walsh JM. Effect of 5-aminosalicylate use on colorectal cancer and dysplasia risk: a systematic review and metaanalysis of observational studies. Am J Gastroenterol 2005;100:1345-1353.

15. Takikawa H, Sakisaka S, Aiso M, et al. Recent status of druginduced liver injury: an analysis of 366 cases between 2002 and 2006. Kanzo 2007;48:517-521. 
16. Abe K, Imaizumi H, Hayashi M, et al. Is the DLST valuable for diagnosis? Kan Tan Sui 2014;68:155-160.

17. Eisenthal A, Eytan K, Brazowski E, Gitstein G, Greenberg R, Skornick Y. Effects of 5-FU on DNA synthesis and cytotoxicity of human lymphocytes induced by IL-2, TGF-beta3 and PGE2. Anticancer Res 2009;29:3925-3930.

18. Yamaguchi M. Drug-induced lymphocyte stimulation test. Medicina 2015;52:327-328.

19. Shimizu H, Arai K, Tang J, Hosoi K, Funayama R. 5-Aminosalicylate intolerance causing exacerbation in pediatric ulcerative colitis. Pediatr Int 2017;59:583-587.

20. Wada S, Kumagai H, Yokoyama K, et al. Mesalazine allergy in a boy with ulcerative colitis: clinical usefulness of mucosal biopsy criteria. Clin J Gastroenterol 2016;9:302-305.

21. Fukushima T, Nakajima K, Henmi H, et al. Desensitization therapy for mesalazine-intolerant patients with inflammatory bowel disease. J Jpn Soc Coloproctol 2014;67:259-262.
22. Solem CA, Loftus EV Jr, Tremaine WJ, Harmsen WS, Zinsmeister AR, Sandborn WJ. Correlation of C-reactive protein with clinical, endoscopic, histologic, and radiographic activity in inflammatory bowel disease. Inflamm Bowel Dis 2005;11:707712.

23. Henriksen M, Jahnsen J, Lygren I, et al. C-reactive protein: a predictive factor and marker of inflammation in inflammatory bowel disease: results from a prospective population-based study. Gut 2008;57:1518-1523.

24. Pichler WJ, Tilch J. The lymphocyte transformation test in the diagnosis of drug hypersensitivity. Allergy 2004;59:809-820.

25. Popple A, Williams J, Maxwell G, Gellatly N, Dearman RJ, Kimber I. The lymphocyte transformation test in allergic contact dermatitis: new opportunities. J Immunotoxicol 2016;13:84-91.

26. Sugihara T, Koda M, Okamoto T, et al. The usefulness of second drug-induced lymphocyte stimulation tests (DLST). Kanzo 2016; 57: 571-576.

27. Fukuda H. Drug-induced lymphocyte stimulation test (DLST). Dr Salon 2012;56:35-39. 